\title{
Higher incidence of perivalvular abscess determines perioperative clinical outcome in patients undergoing surgery for prosthetic valve endocarditis
}

Carolyn Weber ${ }^{1 * \dagger}$ (D, Parwis B. Rahmanian ${ }^{\dagger}$, Melanie Nitsche ${ }^{1}$, Asmae Gassa ${ }^{1}$, Kaveh Eghbalzadeh ${ }^{1}$, Stefanie Hamacher ${ }^{2}$, Julia Merkle ${ }^{1}$, Antje-Christin Deppe ${ }^{1}$, Anton Sabashnikov , Elmar W. Kuhn',

Oliver J. Liakopoulos ${ }^{1}$ and Thorsten Wahlers ${ }^{1}$

\begin{abstract}
Background: Cardiac surgery for prosthetic valve endocarditis (PVE) is associated with substantial mortality. We aimed to analyze 30-day and 1-year outcome in patients undergoing surgery for PVE and sought to identify preoperative risk factors for mortality with special regard to perivalvular infection.

Methods: We retrospectively analyzed data of 418 patients undergoing valve surgery for infective endocarditis between January 2009 and July 2018. After 1:1 propensity matching 158 patients (79 PVE/79 NVE) were analyzed with regard to postoperative 30-day and 1-year outcomes. Univariate and multivariable analyses were performed to identify potential risk factors for mortality.
\end{abstract}

Results: 315 patients (75.4\%) underwent surgery for NVE and 103 (24.6\%) for PVE. After propensity matching groups were comparable with regard to preoperative characteristics, clinical presentation and microbiological findings, except a higher incidence of perivalvular infection in patients with PVE (51.9\%) compared to NVE (26.6\%) ( $p=$ $0.001)$, longer cardiopulmonary bypass (166 [76-130] vs. 97 [71-125] min; $p<0.001)$ and crossclamp time (95 [71$125]$ vs. 68 [55-85] min; $p<0.001)$. Matched patients with PVE showed a 4-fold increased 30-day mortality (20.3\%) in comparison with NVE patients (5.1\%) $(p=0.004)$ and 2 -fold increased 1 -year mortality (PVE 29.1\% Vs. NVE 13.9\%; $p=0.020$ ). Multivariable analysis revealed perivalvular abscess, sepsis, preoperative AKI and PVE as independent risk factors for mortality. Patients with perivalvular abscess had a significantly higher 30-day mortality (17.7\%) compared to patients without perivalvular abscess $(8.0 \%)(p=0.003)$ and a higher rate of perioperative complications (need for postoperative pacemaker implantation, postoperative cerebrovascular events, postoperative AKI). However, perivalvular abscess did not influence 1 -year mortality (20.9\% vs. $22.3 \%$; $p=0.806)$, or long-term complications such as readmission rate or relapse of IE.

Conclusions: Patients undergoing surgery for PVE had a significantly higher 30-day and 1-year mortality compared to NVE. After propensity-matching 30-day mortality was still 4-fold increased in PVE compared to NVE. Patients with perivalvular abscess showed a significantly higher 30-day mortality and perioperative complications, whereas perivalvular abscess seems to have no relevant impact on 1-year mortality, the rate of readmission or relapse of IE.

Keywords: Prosthetic valve endocarditis, PVE, Perivalvular infection, Perivalvular abscess

\footnotetext{
* Correspondence: carolyn.weber@uk-koeln.de

${ }^{\dagger}$ Carolyn Weber and Parwis B. Rahmanian contributed equally to this work

${ }^{1}$ Department of Cardiothoracic Surgery, University of Cologne, Kerpener

Strasse 62, D-50937 Cologne, Germany

Full list of author information is available at the end of the article
}

(c) The Author(s). 2020 Open Access This article is distributed under the terms of the Creative Commons Attribution 4.0 International License (http://creativecommons.org/licenses/by/4.0/), which permits unrestricted use, distribution, and reproduction in any medium, provided you give appropriate credit to the original author(s) and the source, provide a link to the Creative Commons license, and indicate if changes were made. The Creative Commons Public Domain Dedication waiver (http://creativecommons.org/publicdomain/zero/1.0/) applies to the data made available in this article, unless otherwise stated. 


\section{Background}

Among patients undergoing valve operation for infective endocarditis (IE), surgery for prosthetic valve endocarditis (PVE) has been associated with distinct mortality rates in cardiothoracic surgery [1-3]. PVE accounts for $10-30 \%$ of all cases of IE and occurs in 1-6\% of patients with valve prostheses [4-6]. Clinical presentation is often atypical and negative echocardiographic findings are more common, leading to lower sensitivity of the Duke criteria in patients with suspected PVE [7]. Hence, timely diagnosis of endocarditis is more difficult in presence of a prosthetic valve compared with a native valve $[5,8,9]$. One of the reasons, for the higher rate of negative echocardiographic findings in PVE is, that vegetations occur less frequent, whereas periannular extensions are more common, which are more difficult to detect in echocardiography $[4,5]$.

Several factors have been associated with bad prognosis in PVE. Perivalvular infection is of a greater concern in PVE than NVE and occurs in $56-100 \%$ of PVE patients [1, $4,10]$. Previous studies suggested, that perivalvular infection worsens the prognosis, the perioperative mortality and the risk for reinfection or relapse of endocarditis [11, 12]. The extension of the infection beyond the valve annulus leads to technically more demanding operations, requiring radical debridement and reconstruction [10].

Therefore, we aimed to compare 30-day and 1-year outcome after valve surgery for NVE and PVE. Our second objective was to identify potential preoperative risk factors for mortality with special regard to perivalvular infection.

\section{Methods}

\section{Study design}

We performed a retrospective single-center analysis. Relevant clinical data of all consecutive patients undergoing surgery for IE between January 2009 and July 2018 were extracted from our institutional database.

\section{Definition of IE and indication for surgery}

IE was defined according to the recent modified Duke Criteria [13]. Surgery for IE was indicated according to the recent ESC guidelines for the management of infective endocarditis [9] and performed as previously described [14].

\section{Data collection}

Patients' demographics, predisposing risk factors and symptoms at the time of onset of IE, echocardiographic and microbiological findings, perioperative data and relevant clinical outcomes were recorded. IE relevant 30-day and 1-year outcomes were reported for hospital stay and at follow-up, respectively. Follow-up was obtained by review of hospital medical records and interview of the patient's physician. Median duration of the follow-up was 2.05 years [interquartile range (IQR) 0.04-4.69] with a completeness of $76.3 \%$. The follow-up time for survival was measured from the date of operation to either the date of death or the date of the last contact with the patient. The study protocol was approved by the institutional review board (Ethics Committee of the Medical Faculty, University of Cologne, 17-407). Individual informed consent was waived due to the retrospective nature of the collected data.

\section{Reporting mortality}

30-day mortality (day 1-30) was reported as all-cause mortality within the first 30 days after surgery for IE, regardless of the patient's location (at home or health care facility). 1-year mortality was reported as all-cause mortality occurring between day 31 and 365 after initial surgery. Long-term mortality was defined as all-cause mortality after day 365 and Kaplan-Meier analysis was used to test for differences in long-term survival .

\section{Statistical analysis}

Patients' characteristics and pre-operative factors were described using mean values \pm standard deviation (SD), median $[(\mathrm{IQR})]$, or frequencies and percentages as indicated. Depending on data distribution group differences were compared using unpaired t-test, Mann-Whitney U test, Chi-squared test or Fisher's exact test as appropriate. Log-rank test was used to test for differences in long-term mortality between PVE and NVE. A 1:1 propensity score matching was performed to exclude potential confounders between groups with a 0.01 caliper width. This propensity score-based matching procedure resulted in a total number of 158 patients. Matching variables were the following preoperative characteristics that showed statistically significant differences or that were considered clinically significant based on previous research: age, sex, aortic/mitral valve IE, preoperative acute kidney injury (AKI) and Staphylococcus aureus as causative microorganism. Potential risk factors for 30day mortality (day 1-30) were assessed using logistic regression. We decided not to include EuroSCORE into the univariate analysis because EuroSCORE is per definition higher in patients with previous cardiac surgery. After univariate analysis all variables with a $p$-value less than 0.1 (female gender, age $>65$ years, PVE, preoperative AKI, preoperative sepsis, perivalvular abscess, IE with Staphylococcus aureus) were entered into the multivariable model using a forward selection (likelihood ratio, $\mathrm{p}_{\text {in }}=0.05$ ). Results are presented as odds ratio (OR) for 30-day mortality with corresponding $95 \%$ confidence interval $(\mathrm{CI})$ and $p$-value. All reported $p$-values are twosided and considered statistically significant if $\leq 5 \%$. Statistical analyses were performed using SPSS Statistics Version 25 (IBM Corp., Armonk, NY, USA). 


\section{Results}

\section{Preoperative characteristics and risk factors}

Data of 418 patients undergoing surgery for infective endocarditis were retrospectively analyzed. 315 patients (75.4\%) underwent surgery for NVE and 103 patients (24.6\%) for PVE. Table 1 summarizes patients' demographics and preoperative characteristics. PVE patients in the unmatched cohort were significantly older (71.5 [62.0-76.6] vs. 62.7 [49.4-71.6]; $p<0.001)$, showed a higher proportion of female patients being affected $(32.0 \%$ vs. $22.5 \% ; p=0.046)$ and were diagnosed with more preoperative AKI $(67.0 \%$ vs. $54.9 \% ; p=0.031)$. In addition, the manifestation of IE differed between PVE and NVE patients (Table 2). The involvement of the aortic valve was more common in the PVE cohort (78.6\% vs. $52.7 \%$, $p<0.001)$, whereas mitral valve involvement occurred more often in NVE patients $(54.3 \%$ vs. $25.2 \%, \mathrm{p}<0.001)$. Echocardiography revealed vegetations in $82.2 \%$ of NVE and $70.9 \%$ of PVE patients $(p=0.017)$. Inversely, PVE patients were diagnosed with more perivalvular infection. Hence, perivalvular abscess was diagnosed in $60.2 \%$ of the PVE and $27.0 \%$ of the NVE group $(p<0.001)$. Concerning the underlying microorganisms, Coagulase-negative Staphylococci (CoNS) were detected more often in PVE (15.5\% vs. 8.3\%; $p=0.033)$, whereas Streptococcus spp were more prevalent in $\operatorname{NVE}(26.7 \%$ vs. $9.7 \%$; $p<0.001)$. The proportion of Staphylococcus aureus IE was comparable and occurred in PVE with $18.4 \%$ and NVE with $23.5 \%(p=0.285)$. Clinical symptoms were similar among both groups (Table 2). Detailed findings regarding causative microorganisms are depicted in Fig. 1.

After propensity matching there were no statistically significant differences regarding preoperative characteristics, clinical symptoms and manifestation of IE, except a higher rate of perivalvular infection $(51.9 \%$ vs. $26.6 \%, p=0.001)$ (Tables 1 and 2). Due to the complexity of operation, CPB

Table 1 Patients' demographics and preoperative characteristics

\begin{tabular}{|c|c|c|c|c|c|c|c|c|c|c|}
\hline \multirow[b]{3}{*}{ Age } & \multicolumn{5}{|c|}{ ENTIRE COHORT } & \multicolumn{5}{|c|}{ PROPENSITY MATCHED COHORT } \\
\hline & \multicolumn{2}{|c|}{ NVE $(n=315)$} & \multicolumn{2}{|c|}{$\operatorname{PVE}(n=103)$} & \multirow{2}{*}{$\begin{array}{l}\text { P value } \\
<0.001\end{array}$} & \multicolumn{2}{|c|}{ NVE $(n=79)$} & \multicolumn{2}{|c|}{ PVE $(n=79)$} & \multirow{2}{*}{$\frac{P \text { value }}{0.204}$} \\
\hline & 62.7 & {$[49.4-71.6]$} & 71.5 & {$[62.0-76.6]$} & & 65.5 & {$[55.5-72.2]$} & 69.2 & {$[55.5-75.5]$} & \\
\hline Female sex & 71 & $(22.5 \%)$ & 33 & $(32.0 \%)$ & 0.046 & 22 & $(27.8 \%)$ & 19 & $(24.1 \%)$ & 0.586 \\
\hline BMl & 25.5 & {$[23.2-28.1]$} & 26.0 & {$[23.9-28.7]$} & 0.273 & 25.4 & {$[23.7-28.8]$} & 26.1 & {$[23.9-28.7]$} & 0.548 \\
\hline BSA & 1.98 & {$[1.83-2.12]$} & 1.94 & {$[1.74-2.10]$} & 0.238 & 1.97 & \pm 0.21 & 1.97 & \pm 0.24 & 0.349 \\
\hline COPD & 29 & $(9.2 \%)$ & 9 & $(8.7 \%)$ & 0.886 & 10 & $(12.7 \%)$ & 8 & $(10.1 \%)$ & 0.617 \\
\hline Diabetes & 81 & $(25.7 \%)$ & 34 & (33.0\%) & 0.150 & 21 & $(26.6 \%)$ & 25 & $(31.6 \%)$ & 0.484 \\
\hline Peripheral vascular disease & 23 & $(7.3 \%)$ & 12 & $(11.7 \%)$ & 0.167 & 6 & $(7.6 \%)$ & 7 & $(8.9 \%)$ & 0.772 \\
\hline Preoperative AKI & 173 & $(54.9 \%)$ & 69 & $(67.0 \%)$ & 0.031 & 54 & $(68.4 \%)$ & 52 & $(65.8 \%)$ & 0.735 \\
\hline Preoperative dialysis & 30 & $(9.5 \%)$ & 13 & $(12.6 \%)$ & 0.889 & 6 & $(7.6 \%)$ & 13 & $(16.5 \%)$ & 0.176 \\
\hline Coronary artery disease & 80 & (25.4\%) & 36 & $(35.5 \%)$ & 0.060 & 23 & $(29.1 \%)$ & 22 & $(27.8 \%)$ & 0.860 \\
\hline Prior PCl & 18 & $(5.7 \%)$ & 14 & $(13.6 \%)$ & 0.054 & 4 & $(5.1 \%)$ & 8 & $(10.1 \%)$ & 0.124 \\
\hline Immunosuppression & 5 & $(1.6 \%)$ & 2 & $(1.9 \%)$ & 0.811 & 1 & $(1.3 \%)$ & 1 & $(1.3 \%)$ & 1.000 \\
\hline HIV & 9 & $(2.9 \%)$ & 1 & $(1.0 \%)$ & 0.233 & 0 & $(0 \%)$ & 1 & $(1.3 \%)$ & 0.238 \\
\hline Alcohol abuse & 37 & $(11.7 \%)$ & 4 & $(3.9 \%)$ & 0.020 & 8 & $(10.1 \%)$ & 3 & $(3.8 \%)$ & 0.118 \\
\hline Intravenous drug abuse & 22 & $(7.0 \%)$ & 6 & $(5.8 \%)$ & 0.683 & 5 & $(6.3 \%)$ & 6 & $(7.6 \%)$ & 0.755 \\
\hline History of neoplasm & 33 & $(10.5 \%)$ & 10 & $(9.7 \%)$ & 0.824 & 12 & $(15.2 \%)$ & 6 & $(7.6 \%)$ & 0.133 \\
\hline \multicolumn{11}{|l|}{ LVEF } \\
\hline$<30 \%$ & 7 & $(2.2 \%)$ & 2 & $(1.9 \%)$ & 0.865 & 1 & $(1.3 \%)$ & 2 & $(2.5 \%)$ & 0.559 \\
\hline $30-50 \%$ & 63 & $(20 \%)$ & 30 & (29.1\%) & 0.532 & 16 & $(20.3 \%)$ & 23 & (29.1\%) & 0.268 \\
\hline$>50 \%$ & 238 & $(75.6 \%)$ & 70 & $(70.0 \%)$ & 0.129 & 62 & $(78.5 \%)$ & 53 & $(67.1 \%)$ & 0.108 \\
\hline \multicolumn{11}{|l|}{ NYHA class } \\
\hline $1+\|$ & 55 & $(17.5 \%)$ & 19 & $(18.4 \%)$ & 0.925 & 20 & $(25.3 \%)$ & 14 & (17.7\%) & 0.245 \\
\hline$I I I+I V$ & 251 & (80.0\%) & 80 & $(77.7 \%)$ & 0.662 & 59 & (74.7\%) & 63 & (79.7\%) & 0.448 \\
\hline Log. EuroSCORE & 7.6 & [4.4-18.1] & 9.8 & {$[22.3-36.2]$} & $<0.001$ & 7.9 & {$[4.1-16.3]$} & 17.5 & [7.9-33.7] & $<0.001$ \\
\hline EuroSCORE II & 7.0 & [5.0-10.0] & 11.0 & [8.0-13.0] & $<0.001$ & 7.0 & [5.0-9.0] & 10.0 & [7.0-12.3] & $<0.001$ \\
\hline
\end{tabular}

Data presented as mean \pm standard deviation, number (percent) or median [IQR], respectively. AKI, acute kidney injury; $B M I$, body mass index; $B S A$, body surface area; COPD, chronic obstructive pulmonary disease; HIV, human immunodeficiency virus; IQR, interquartile range; NYHA, New York Heart Association; PCI, percutaneous coronary intervention; TIA, transient ischemic attack 
Table 2 Manifestation of IE according to the modified Duke Criteria

\begin{tabular}{|c|c|c|c|c|c|c|c|c|c|c|}
\hline \multirow{3}{*}{ MICROBIOLOGY } & \multicolumn{5}{|c|}{ ENTIRE COHORT } & \multicolumn{5}{|c|}{ PROPENSITY MATCHED COHORT } \\
\hline & \multicolumn{2}{|c|}{$\operatorname{NVE}(n=315)$} & \multicolumn{2}{|c|}{ PVE $(n=103)$} & \multirow[t]{2}{*}{$P$ value } & \multicolumn{2}{|c|}{$\operatorname{NVE}(n=315)$} & \multicolumn{2}{|c|}{ PVE $(n=103)$} & \multirow[t]{2}{*}{$P$ value } \\
\hline & & & & & & & & & & \\
\hline Positive Blood culture & 263 & (83.5\%) & 77 & $(74.8 \%)$ & 0.279 & 65 & $(82.3 \%)$ & 58 & $(73.4 \%)$ & 0.505 \\
\hline Streptococcus spp & 84 & $(26.7 \%)$ & 10 & $(9.7 \%)$ & $<0.001$ & 20 & $(25.3 \%)$ & 8 & $(10.1 \%)$ & 0.011 \\
\hline Staphylococcus spp & 98 & (31.1\%) & 35 & (34.0\%) & 0.587 & 20 & $(25.3 \%)$ & 24 & $(30.4 \%)$ & 0.478 \\
\hline Staph. aureus & 74 & $(23.5 \%)$ & 19 & $(18.4 \%)$ & 0.285 & 16 & (20.3\%) & 15 & $(19.0 \%)$ & 0.841 \\
\hline CONS & 26 & $(8.3 \%)$ & 16 & $(15.5 \%)$ & 0.033 & 5 & $(6.3 \%)$ & 9 & $(11.4 \%)$ & 0.263 \\
\hline Enterococcus spp & 44 & $(14.0 \%)$ & 17 & $(16.5 \%)$ & 0.527 & 15 & $(19.0 \%)$ & 16 & $(20.3 \%)$ & 0.841 \\
\hline \multicolumn{11}{|l|}{ ECHOCARDIOGRAPHY } \\
\hline Vegetation & 259 & $(82.2 \%)$ & 73 & $(70.9 \%)$ & 0.017 & 65 & (82.3\%) & 59 & $(74.7 \%)$ & 0.307 \\
\hline Vegetation length (cm) & 1.6 & {$[1.1-2.0]$} & 1.2 & {$[0.8-1.7]$} & 0.009 & 1.5 & \pm 0.6 & 1.3 & \pm 0.7 & 0.357 \\
\hline \multicolumn{11}{|l|}{ Leftsided IE } \\
\hline Aortic valve & 166 & $(52.7 \%)$ & 81 & (78.6\%) & $<0.001$ & 52 & $(65.8 \%)$ & 58 & $(73.4 \%)$ & 0.299 \\
\hline Mitral valve & 171 & $(54.3 \%)$ & 26 & $(25.2 \%)$ & $<0.001$ & 23 & (29.1\%) & 26 & $(32.9 \%)$ & 0.606 \\
\hline \multicolumn{11}{|l|}{ Rightsided IE } \\
\hline Tricuspid valve & 21 & $(6.7 \%)$ & 1 & $(1.0 \%)$ & 0.046 & 6 & $(7.6 \%)$ & 1 & $(1.3 \%)$ & 0.053 \\
\hline Pulmonary valve & 1 & $(0.3 \%)$ & 1 & $(1.0 \%)$ & 0.439 & 0 & $(0 \%)$ & 1 & $(1.3 \%)$ & 0.238 \\
\hline \multicolumn{11}{|l|}{ Perivalvular infection } \\
\hline Perivalvular abscess & 85 & $(27.0 \%)$ & 62 & $(60.2 \%)$ & $<0.001$ & 21 & $(26.6 \%)$ & 41 & $(51.9 \%)$ & 0.001 \\
\hline Perforation & 83 & $(26.3 \%)$ & 21 & $(20.4 \%)$ & 0.224 & 10 & $(12.7 \%)$ & 19 & $(24.1 \%)$ & 0.064 \\
\hline Fistula & 1 & $(0.3 \%)$ & 13 & $(12.6 \%)$ & $<0.001$ & 1 & $(1.3 \%)$ & 10 & $(12.7 \%)$ & 0.005 \\
\hline \multicolumn{11}{|l|}{ SYMPTOMS } \\
\hline Fever & 201 & $(63.8 \%)$ & 72 & (69.9\%) & 0.259 & 60 & $(75.9 \%)$ & 55 & $(69.6 \%)$ & 0.371 \\
\hline Sepsis & 233 & $(74.0 \%)$ & 68 & $(66.0 \%)$ & 0.119 & 41 & $(51.9 \%)$ & 38 & $(48.1 \%)$ & 0.633 \\
\hline IE-related neurologic complications & 99 & $(31.4 \%)$ & 28 & $(27.2 \%)$ & 0.416 & 23 & $(29.1 \%)$ & 22 & $(27.8 \%)$ & 0.860 \\
\hline$T I A$ & 13 & $(4.1 \%)$ & 1 & $(1.0 \%)$ & 0.122 & 3 & $(3.8 \%)$ & 0 & $(0 \%)$ & 0.305 \\
\hline Stroke & 47 & (14.9\%) & 17 & $(16.5 \%)$ & 0.698 & 11 & (13.9\%) & 14 & $(17.7 \%)$ & 0.513 \\
\hline Intracranial bleeding & 5 & $(1.6 \%)$ & 3 & $(2.9 \%)$ & 0.394 & 1 & $(1.3 \%)$ & 3 & $(3.8 \%)$ & 0.311 \\
\hline Other & 33 & $(10.5 \%)$ & 7 & $(6.8 \%)$ & 0.270 & 8 & (10.1\%) & 5 & $(6.3 \%)$ & 0.385 \\
\hline Septic embolism & 115 & (36.5\%) & 29 & $(28.2 \%)$ & 0.188 & 25 & (31.6\%) & 24 & $(30.4 \%)$ & 0.239 \\
\hline Cardiogenic shock & 46 & $(14.6 \%)$ & 9 & $(8.7 \%)$ & 0.126 & 7 & (8.9\%) & 8 & $(10.1 \%)$ & 0.786 \\
\hline
\end{tabular}

Data presented as mean \pm standard deviation, number (percent) or median [IQR], respectively. $d$, days; IE infective endocarditis; IQR, interquartile range; TIA, transient ischemic attack

and crossclamp time were significantly longer in patients with PVE (CPB time: 166 [76-130] vs. 97 [71-125] min; $p<0.001$; crossclamp time: 95 [71-125] vs. 68 [55-85] min; $\mathrm{p}<0.001$ ) (Additional file 1: Table S1).

\section{0-day and 1-year clinical outcomes}

30-day and 1-year clinical outcomes are depicted in Table 3. With regard to 30-day outcome, PVE was associated with a significantly higher 30-day mortality. After propensitymatching 30-day mortality was still 4-fold increased in PVE compared to NVE $(20.3 \%$ vs. $5.1 \%$; $p=0.004)$. Concerning the incidence of postoperative complications, matched cohorts were comparable except a longer time of ventilation
(28.3 [15-113] vs. $16.8[12-46]$ hours; $p=0.017)$, longer ICU (5.0 [2.0-12.0] vs. 3.0 [1.0-6.0] days; $p=0.042)$ and hospital stay (15.0 [11.0-20.0] vs. 12.0 [7.0-17.0] days; $p=$ $0.015)$ in the PVE cohort.

In addition, 1-year mortality was significantly higher in PVE $(29.1 \%)$ compared to NVE $(13.9 \%, p=0.020)$ in the matched cohort. Kaplan-Meier survival analysis revealed a significantly decreased long-term survival of patients undergoing surgery for PVE compared to NVE in the unmatched cohort (log-rank $p=0.019$; Fig. 2a). After propensity matching no significant difference with regard to long-term survival could be found $(\log$-rank $p=$ 0.174; Fig. 2b). 


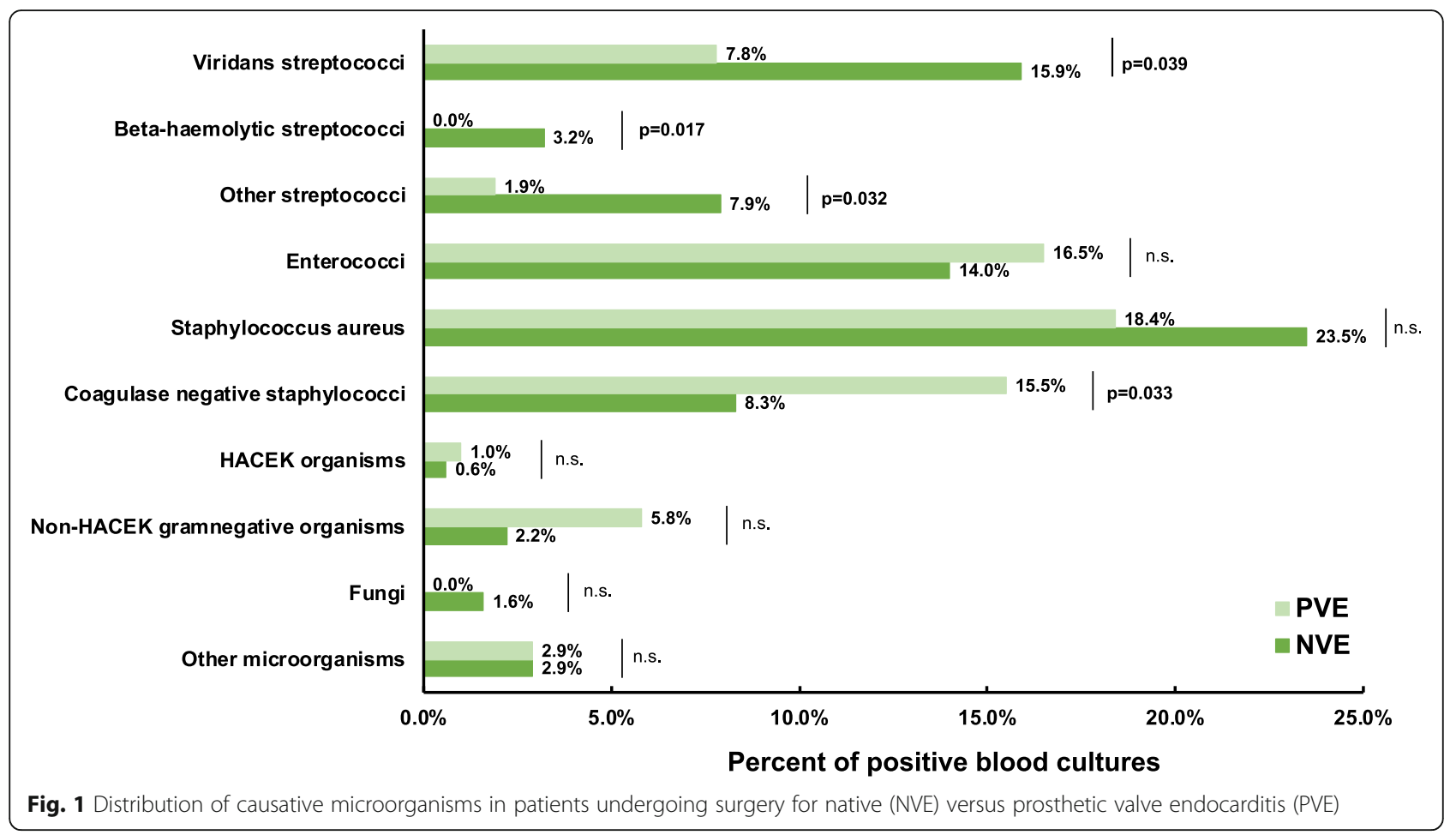

Table 3 30-day and 1-year clinical outcomes

\begin{tabular}{|c|c|c|c|c|c|c|c|c|c|c|}
\hline & ENTIF & COHORT & & & & PROF & SITY MATCH & $\mathrm{COH}$ & & \\
\hline & NVE & $=315)$ & PVE & $=103)$ & $P$ value & NVE & $=315)$ & $\begin{array}{l}\text { PVE } \\
(\mathrm{n}=\end{array}$ & & $P$ value \\
\hline 30-day mortality & 26 & $(8.3 \%)$ & 22 & $(21.4 \%)$ & $<0.001$ & 4 & $(5.1 \%)$ & 16 & $(20.3 \%)$ & 0.004 \\
\hline Myocardial infarction & 1 & $(0.3 \%)$ & 1 & $(1.0 \%)$ & 0.439 & 0 & $(0 \%)$ & 1 & $(1.3 \%)$ & 0.238 \\
\hline New pacemaker * & 23 & $(7.3 \%)$ & 20 & $(19.4 \%)$ & $<0.001$ & 8 & (10.1\%) & 11 & (13.9\%) & 0.463 \\
\hline $\begin{array}{l}\text { New postoperative } \\
\text { cerebrovascular events }\end{array}$ & 15 & $(4.8 \%)$ & 8 & $(7.8 \%)$ & 0.252 & 6 & $(7.6 \%)$ & 4 & $(5.1 \%)$ & 0.499 \\
\hline Stroke & 14 & $(4.4 \%)$ & 5 & $(4.9 \%)$ & 0.862 & 6 & $(7.6 \%)$ & 3 & $(3.8 \%)$ & 0.294 \\
\hline Intracranial bleeding & 1 & $(0.3 \%)$ & 3 & $(2.9 \%)$ & 0.077 & 0 & (0\%) & 1 & $(1.3 \%)$ & 0.238 \\
\hline Postoperative AKI & 107 & (34.0\%) & 47 & $(45.6 \%)$ & 0.035 & 33 & (41.8\%) & 32 & $(40.5 \%)$ & 0.872 \\
\hline Re-exploration for bleeding & 45 & $(14.3 \%)$ & 26 & $(25.2 \%)$ & 0.009 & 10 & $(12.7 \%)$ & 17 & $(21.5 \%)$ & 0.129 \\
\hline Tracheostomy & 38 & $(12.1 \%)$ & 21 & $(20.4 \%)$ & 0.036 & 10 & $(12.7 \%)$ & 15 & $(19.0 \%)$ & 0.276 \\
\hline Time of ventilation (h) & 18.2 & [11-68] & 32.8 & [17-120] & $<0.001$ & 16.8 & [12-46] & 28.3 & [15-113] & 0.017 \\
\hline ICU stay $(\mathrm{d})$ & 4.0 & {$[2.0-8.0]$} & 5.0 & {$[3.0-13.0]$} & 0.009 & 3.0 & {$[1.0-6.0]$} & 5.0 & {$[2.0-12.0]$} & 0.042 \\
\hline Hospital stay $(\mathrm{d})$ & 12.0 & {$[8.0-16.0]$} & 14.5 & {$[10.0-20.0]$} & 0.007 & 12.0 & {$[7.0-17.0]$} & 15.0 & {$[11.0-20.0]$} & 0.015 \\
\hline 1-YEAR OUTCOME & & & & & & & & & & \\
\hline 1-year mortality & 53 & $(16.8 \%)$ & 34 & $(33.0 \%)$ & $<0.001$ & 11 & (13.9\%) & 23 & $(29.1 \%)$ & 0.020 \\
\hline Re-admission to hospital & 94 & $(29.8 \%)$ & 33 & $(32.0 \%)$ & 0.478 & 31 & (39.2\%) & 23 & $(29.1 \%)$ & 0.835 \\
\hline Relapse of endocarditis & 10 & $(3.2 \%)$ & 3 & $(2.9 \%)$ & 0.938 & 3 & $(3.8 \%)$ & 2 & $(2.5 \%)$ & 1.000 \\
\hline New pacemaker* & 5 & $(1.6 \%)$ & 4 & $(3.9 \%)$ & 0.177 & 2 & $(2.5 \%)$ & 1 & $(1.3 \%)$ & 0.792 \\
\hline AKI during follow-up & 23 & $(7.3 \%)$ & 13 & $(12.6 \%)$ & 0.071 & 11 & (13.9\%) & 8 & $(10.1 \%)$ & 0.883 \\
\hline
\end{tabular}

Data presented as mean \pm standard deviation, number (percent) or median [IQR], respectively. AKI, acute kidney injury; ICU, intensive care unit; IQR, interquartile range; TIA, transient ischemic attack; ${ }^{*}$ for AV-higher grade 

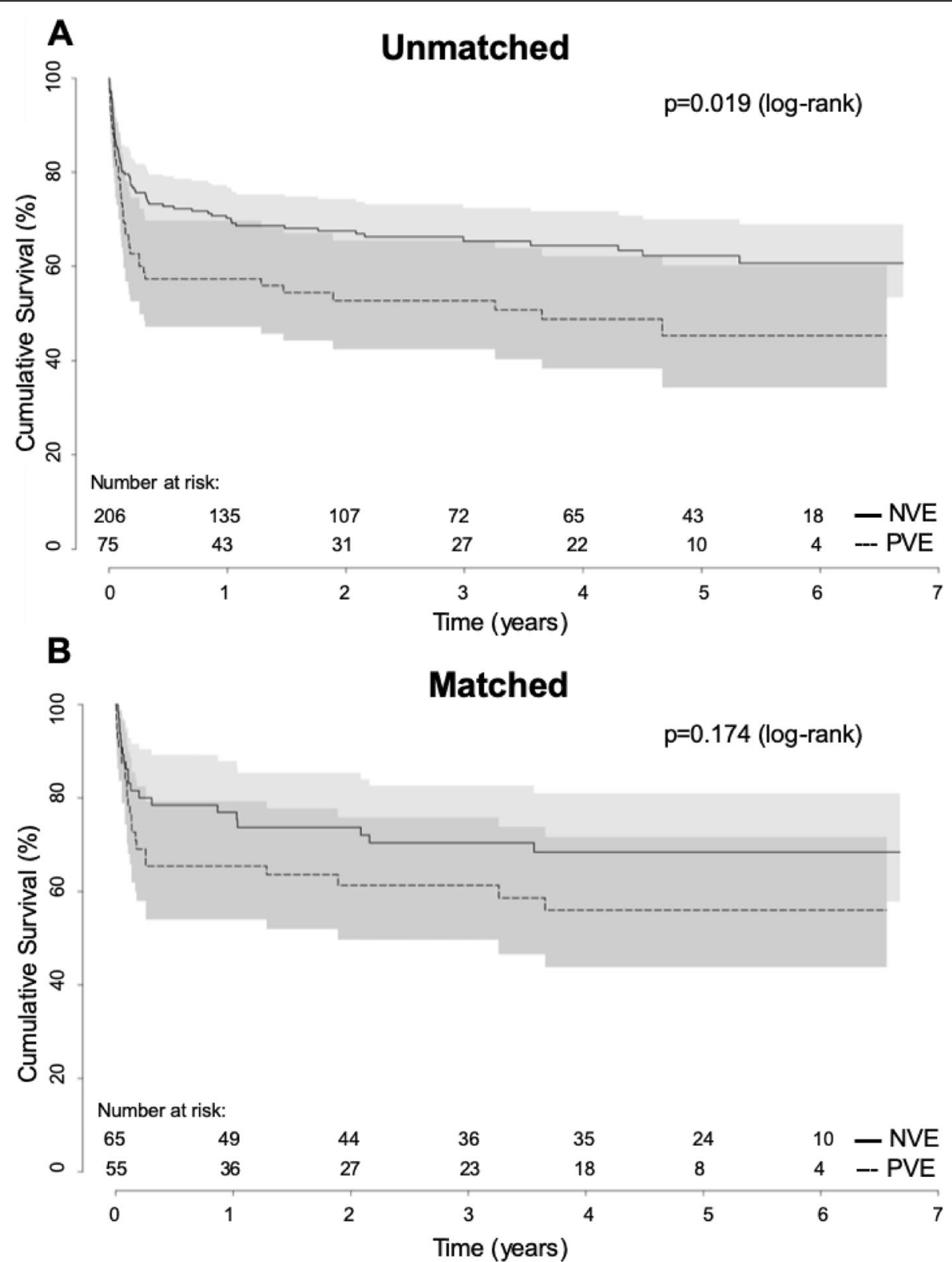

Fig. 2 Long-term survival of patients undergoing cardiac surgery for native (NVE) versus prosthetic valve endocarditis (PVE). a Higher long-term mortality of patients with PVE compared to NVE. b After propensity-matching for relevant preoperative risk factors comparable long-term survival between NVE and PVE patients

\section{Factors associated with mortality}

Univariate analysis revealed the following preoperative variables as factors associated with 30-day mortality: preoperative AKI, PVE, preoperative sepsis, perivalvular abscess, IE with Staphylococcus aureus (Additional file 1: Table S2).Stepwise multivariable logistic regression analysis revealed perivalvular abscess (OR 1.864 [1.0023.465]; $p=0.049$ ), preoperative AKI (OR 2.720 [1.3075.657]; $p=0.007$ ) and preoperative sepsis (OR 2.281 [1.123-4.636]; $p=0.023$ ) as independent risk factors for 30-day mortality. In addition, PVE was independently predictive for 30-day mortality (OR 2.699 [1.496-4.871]; $p=0.001$ ) (Table 4).

\section{Patients with perivalvular abscess}

Patients with perivalvular abscess showed no statistically significant differences regarding preoperative characteristics except a higher rate of peripheral vascular disease (12.2\% vs. $6.2 \%, p=0.033)$. Regarding the manifestation of IE, we found no difference in the distribution of underlying causative microorganisms. But patients with perivalvular abscess were diagnosed more with IE of the 
Table 4 Multivariable analysis of risk factors associated with 30day mortality in patients undergoing surgery for IE INDEPENDENT RISK FACTORS FOR 30-DAY MORTALITY

\begin{tabular}{llll}
\hline & OR & $95 \% \mathrm{Cl}$ & P value \\
\hline PVE & 2.699 & $1.496-4.871$ & 0.001 \\
Preoperative AKI & 2.720 & $1.307-5.657$ & 0.007 \\
Preoperative sepsis & 2.281 & $1.123-4.636$ & 0.023 \\
Perivalvular abscess & 1.864 & $1.002-3.465$ & 0.049 \\
\hline
\end{tabular}

$C l$, confidence intervall; IE infective endocarditis; $O R$, odds ratio

aortic valve $(76.4 \%$ vs. $49.5 \%$; $p<0.001)$ whereas patients without perivalvular abscess showed more mitral valve IE $(56.0 \%$ vs. $33.8 \%$; $<<0.001)$. Operation, $\mathrm{CPB}$ and crossclamp time were significantly longer in patients with perivalvular abscess due to the higher complexity of operation.

Patients with perivalvular abscess showed an impaired perioperative outcome with a significantly higher 30-day mortality $(17.7 \%$ vs. $8.0 \% ; p=0.003)$, a higher need for postoperative pacemaker implantation for higher AVgrade $(15.5 \%$ vs. $7.3 \% ; p=0.007)$, an increased rate of postoperative cerebrovascular events $(8.8 \%$ vs. $4.0 \% ; p=$ $0.042)$ and postoperative AKI $(45.6 \%$ vs. $33.1 \% ; p=$ 0.012 ). However, perivalvular abscess seems not to influence 1 -year mortality (20.9\% vs. $22.3 \%$; $p=806)$ and postoperative long-term complications, such as readmission to hospital, relapse of IE or AKI during the followup (Table 5).

\section{Discussion}

\section{Incidence of PVE}

Although the incidence of PVE is relatively low, there is an increasing number of patients with PVE [15]. PVE occurs in 1-6\% of patients with valve prostheses [4-6] and accounted for over $20 \%$ of all IE cases in a prospective, multicenter, international registry, reflecting a considerably higher proportion of PVE compared to earlier reports [4]. The proportion of PVE to NVE is comparable with our data, including $24.6 \%$ of PVE cases in our cohort of patients undergoing surgery for IE.

\section{Postoperative outcome of patients undergoing surgery for PVE compared to NVE}

Our retrospective analysis revealed PVE as independent risk factor for mortality. PVE patients of our cohort had a significantly higher 30 -day mortality compared to NVE patients $(21.4 \%$ vs. $8.3 \% ; p<0.001)$. These findings were also reported by Romano et al. who found a higher inhospital mortality in their PVE group, compared to their NVE group (24.2\% vs. $6.6 \%, p<0.0001)$ [2]. Even after propensity-matching, we still observed a 4-fold increased 30-day mortality in PVE compared to NVE.
Operation for PVE in comparison with NVE has been linked to worse outcomes and the 30-day mortality remains high. Our data demonstrate that the higher mortality of patients undergoing surgery PVE compared to NVE occurs in the early postoperative phase and up to one year postoperatively. Afterwards the Kaplan Meier curve shows parallel slopes, thus a similar long-term survival beyond the first year after surgery. This is in line with findings of Alonso-Valle et al., who found highest mortality rates during the first 3 months. After this period the survival of the patients remained stable [6]. Likewise, Manne et al. found a significantly higher 30day mortality $(13 \%$ vs $5.6 \%, p<0.01)$ in patients with PVE compared to NVE, but long-term survival was not significantly different (35\% vs. $29 \%, p=0.19$ ) [16], which is similar in our study. In addition, data of the Cleveland Clinic suggest, that early mortality is significantly higher in patients with PVE, whereas 1-year survival is comparable [16]. This is in line with the findings of Edlin et al., reporting on similar long-term survival in patients with PVE compared to NVE [15].

Several factors have been associated with the increased mortality in PVE including increased age [17], female sex [18], congestive heart failure [19], staphylococcal infection [4] and renal dysfunction [20]. In several other previous studies, periannular complications were also associated with significantly higher inhospital mortality $[1,4,6,18]$. In line with this, our multivariate analysis revealed PVE, preoperative AKI, preoperative sepsis and perivalvular abscess as independent risk factors for mortality.

\section{Perivalvular infection}

In our cohort we could detect significantly more perivalvular abscesses in $60.2 \%$ of PVE compared to $27.0 \%$ of NVE patients $(p<0.001)$. This is in line with other studies reporting on periannular extensions in 56-100\% of patients with PVE [10]. Due to frequent presence of perivalvular infection, surgery for PVE is technically more demanding than for NVE, as it requires radical debridement and complex surgical intervention $[8,10]$. This is reflected in the longer CPB and crossclamp times in our PVE group, which might be a surrogate parameter for the complexity of procedure. In several previous studies, periannular complications were associated with significantly higher in-hospital mortality $[1,4,6,18,21]$. In accordance, we found a 30-day mortality rate of $17.7 \%$ in patients with perivalvular abscess, which was significantly higher compared to patients without perivalvular abscess (8.0\%) $(p=0.003)$. Besides the higher 30-day mortality, patients with perivalvular abscess suffered from more postoperative complications, such as the need of postoperative pacemaker implantation, new postoperative cerebrovascular events and postoperative 
Table 5 Characteristics and outcomes of patients with and without perivalvular abscess

\begin{tabular}{|c|c|c|c|}
\hline & $\begin{array}{c}\text { - Perivalvular } \\
\text { abscess } \\
(n=275)\end{array}$ & $\begin{array}{c}+ \text { Perivalvular } \\
\text { abscess } \\
(n=148)\end{array}$ & $P$ value \\
\hline Age & $64.3[50.6-72.9]$ & $65.0[50.3-74.0]$ & 0.778 \\
\hline Female sex & $61(22.3 \%)$ & $43(29.1 \%)$ & 0.122 \\
\hline BMl & $25.8[23.5-28.3]$ & $25.4[23.4-28.7]$ & 0.597 \\
\hline BSA & $1.98[1.83-2.12]$ & $1.96[1.74-1.96]$ & 0.309 \\
\hline COPD & $26(9.5 \%)$ & $14(9.5 \%)$ & 0.999 \\
\hline Diabetes & $78(28.4 \%)$ & 39 (26.4\%) & 0.659 \\
\hline Peripheral vascular disease & $17(6.2 \%)$ & $18(12.2 \%)$ & 0.033 \\
\hline Preoperative AKI & $156(56.7 \%)$ & $90(60.8 \%)$ & 0.417 \\
\hline Coronary artery disease & $71(25.8 \%)$ & $46(31.1 \%)$ & 0.248 \\
\hline Immunosuppression & $3(1.1 \%)$ & $4(2.7 \%)$ & 0.229 \\
\hline HIV & $6(2.2 \%)$ & $4(2.7 \%)$ & 0.739 \\
\hline Alcohol abuse & $26(9.5 \%)$ & $15(10.1 \%)$ & 0.821 \\
\hline Intravenous drug abuse & $19(6.9 \%)$ & $9(6.1 \%)$ & 0.774 \\
\hline History of neoplasm & $27(9.8 \%)$ & $16(10.8 \%)$ & 0.747 \\
\hline \multicolumn{4}{|l|}{ LVEF } \\
\hline$<30 \%$ & $4(1.5 \%)$ & $5(3.4 \%)$ & 0.201 \\
\hline $30-50 \%$ & $61(22.8 \%)$ & $33(22.4 \%)$ & 0.942 \\
\hline$>50 \%$ & $203(75.7 \%)$ & 109 (74.1\%) & 0.809 \\
\hline \multicolumn{4}{|l|}{ MICROBIOLOGY } \\
\hline Positive Blood culture & $224(89.6 \%)$ & 119 (84.4\%) & 0.132 \\
\hline Streptococcus spp. & 69 (25.1\%) & $26(17.6 \%)$ & 0.077 \\
\hline Staphylococcus spp. & $83(30.2 \%)$ & $51(34.5 \%)$ & 0.367 \\
\hline Staph aureus & $61(22.2 \%)$ & $33(22.3 \%)$ & 0.978 \\
\hline CoNS & $23(8.4 \%)$ & $19(12.8 \%)$ & 0.142 \\
\hline Enterococcus spp. & $39(14.2 \%)$ & $22(14.9 \%)$ & 0.849 \\
\hline \multicolumn{4}{|l|}{ ECHOCARDIOGRAPHY } \\
\hline Vegetation & $226(82.5 \%)$ & $110(74.8 \%)$ & 0.062 \\
\hline \multicolumn{4}{|l|}{ Leftsided IE } \\
\hline Aortic valve & $136(49.5 \%)$ & $113(76.4 \%)$ & $<0.001$ \\
\hline Mitral valve & $154(56.0 \%)$ & $50(33.8 \%)$ & $<0.001$ \\
\hline \multicolumn{4}{|l|}{ Rightsided IE } \\
\hline Tricuspid valve & $19(6.9 \%)$ & $4(2.7 \%)$ & 0.069 \\
\hline Pulmonary valve & $1(0.7 \%)$ & $1(0.4 \%)$ & 0.663 \\
\hline \multicolumn{4}{|l|}{ SYMPTOMS } \\
\hline Fever & $169(61.5 \%)$ & 107 (72.3\%) & 0.026 \\
\hline Sepsis & $136(49.5 \%)$ & $84(56.8 \%)$ & 0.152 \\
\hline IE-related neurologic complications & $85(30.9 \%)$ & $43(29.1 \%)$ & 0.692 \\
\hline Septic embolism & 99 (36.1\%) & $47(32.2 \%)$ & 0.658 \\
\hline Cardiogenic shock & $31(11.3 \%)$ & $25(16.9 \%)$ & 0.104 \\
\hline \multicolumn{4}{|l|}{ OPERATION } \\
\hline Operation time (min) & 195 [155-245] & 230 [178-314] & $<0.001$ \\
\hline CPB time (min) & 106 [83-142] & 138 [100-182] & $<0.001$ \\
\hline Crossclamp time (min) & 69 [53-95] & 87 [67-115] & $<0.001$ \\
\hline
\end{tabular}


Table 5 Characteristics and outcomes of patients with and without perivalvular abscess (Continued)

\begin{tabular}{|c|c|c|c|}
\hline & $\begin{array}{c}\text { - Perivalvular } \\
\text { abscess } \\
(n=275)\end{array}$ & $\begin{array}{c}+ \text { Perivalvular } \\
\text { abscess } \\
(n=148)\end{array}$ & $P$ value \\
\hline \multicolumn{4}{|l|}{ 30-DAY OUTCOME } \\
\hline 30-day mortality & $22(8.0 \%)$ & $26(17.7 \%)$ & 0.003 \\
\hline Myocardial infarction & $1(0.4 \%)$ & $1(0.7 \%)$ & 0.663 \\
\hline New pacemaker * & $20(7.3 \%)$ & $23(15.5 \%)$ & 0.007 \\
\hline New postoperative cerebrovascular events & $11(4.0 \%)$ & $13(8.8 \%)$ & 0.042 \\
\hline Postoperative AKI & $91(33.1 \%)$ & $67(45.6 \%)$ & 0.012 \\
\hline Re-exploration for bleeding & $43(15.7 \%)$ & $28(18.9 \%)$ & 0.398 \\
\hline Tracheostomy & $33(12.0 \%)$ & $26(17.7 \%)$ & 0.108 \\
\hline Time of ventilation ( $h$ ) & 19.8 [12.0-68.9] & $26.6[12.3-117.6]$ & 0.118 \\
\hline ICU stay $(d)$ & $4.0[2.0-8.0]$ & $5.0[2.0-10.0]$ & 0.103 \\
\hline Hospital stay $(\mathrm{d})$ & $12.0[9.0-17.0]$ & $12.0[9.0-17.0]$ & 0.992 \\
\hline \multicolumn{4}{|l|}{ 1-YEAR OUTCOME } \\
\hline 1-year mortality & $35(22.3 \%)$ & $18(20.9 \%)$ & 0.806 \\
\hline Re-admission to hospital & $86(58.5 \%)$ & $44(55.0 \%)$ & 0.610 \\
\hline Relapse of endocarditis & $10(6.9 \%)$ & $4(5.3 \%)$ & 0.631 \\
\hline AKI during follow-up & $23(15.9 \%)$ & $14(18.2 \%)$ & 0.659 \\
\hline
\end{tabular}

Data presented as number (percent) or median [IQR], respectively. AKI, acute kidney injury; BMI, body mass index; $B S A$, body surface area; COPD, chronic obstructive pulmonary disease; HIV, human immunodeficiency virus; ICU, intensive care unit; IE, infective endocarditis; IQR, interquartile range; ${ }^{*}$ for higher AV-grade

AKI. Our data suggest, that perivalvular abscess influences 30-day mortality and perioperative complications, but has no relevant impact on 1-year mortality or longterm complications in terms of readmission to hospital, relapse of IE or AKI during the follow-up. Therefore, we suppose that the difference in survival during the first year after surgery for PVE and NVE might be attributed to the higher prevalence of perivalvular abscess in PVE patients, which is associated with a higher rate of 30-day mortality and perioperative complications, but has no impact on 1-year mortality, readmission rate or relapse of IE.

\section{Impaired timely diagnosis of PVE}

Timely diagnosis of PVE can be difficult as clinical presentation is frequently atypical and results of blood cultures and echocardiography are more often negative, leading to lower sensitivity of the Duke criteria [7-9]. This is supported by our data, showing a significantly lower rate of vegetations in echocardiography for patients with PVE (70.9\% vs. $82.2 \% ; p=0.017)$. Hence, we suggest that early detection and diagnosis of PVE is essential to prevent an increase of perivalvular destruction. Several studies have investigated the sensitivity and specificity of PET/CT or SPECT/CT imaging in patients with suspected IE $[22,23]$. PET-CT increased the sensitivity and specificity of the modified Duke criteria from 70 to $95 \%$, reducing the number of patients with „possible IE “from 56 to 32\% [22, 23]. A combination of TTE, TEE and CT can increase the sensitivity for the detection of valvular and perivalvular complications in IE [11]. Therefore, early detection of perivalvular lesions by using CT imaging should be considered in suspected IE as implemented in the recent ESC guidelines for the management of IE [9].

\section{Distribution of causative microorganisms}

Besides more perivalvular infection, the spectrum of microorganisms that cause PVE seems to differ from those detected in NVE. Several studies reported, that Staphylococci were more frequent in PVE, with a predominance of Staphylococcus aureus or CoNS [4, 24, 25]. Previous studies revealed, that IE due to Staphylococcus aureus is characterized by aggressive disease with increased risk of embolism, stroke and mortality [26]. However, the incidence of Staphylococcus aureus IE was comparable between PVE and NVE patients in our cohort. Nevertheless, we included infection with Staphylococcus aureus as matching variable in our propensity matching. Although we could not find a difference in the incidence of Staphylococcus aureus IE, patients with PVE were diagnosed more often with CoNS compared to NVE patients. As ubiquitous skin commensals, CoNS are often associated with health-care contact and invasive procedures [7]. They colonise indwelling lines and devices and are the most common isolate in early prosthetic valve endocarditis $[6,7,25]$. 
Staphylococci and gram-negative microorganisms have been suspected to occur as nosocomial perioperative infection during a period when the prosthetic valve is not completely endothelialized [5]. Therefore strict hygiene during health-care contact and invasive procedures in patients with prosthetic valves is essential.

\section{Limitations}

Our study has several limitations that need to be considered for the interpretation of the results. First, the retrospective design and analysis of a limited number of patients from a single center institution reduces the generalizability. In addition, a powerful multivariable analysis of all possible predictors was restricted by the total patient number. Moreover, although we attempted to correct for confounders between groups by propensity matching, there might still remain differences between groups. Finally, we included a very recent patients' cohort, therefore the 1-year follow-up is not completed in all patients. Nevertheless, despite the limited sample size, our study still comprises a relatively large IE cohort compared to the recent literature and provides meaningful results due to propensity matching.

\section{Conclusions}

Considering patients with similar risk profiles, our analysis revealed PVE as an independent risk factor for mortality. PVE was associated with significantly higher 30day and 1-year mortality compared to NVE. After propensity-matching 30-day mortality was still 4-fold increased in PVE compared to NVE.

The higher incidence of perivalvular abscess seems to determine clinical outcome. Patients with perivalvular abscess showed a significantly higher 30-day mortality and perioperative complications, whereas perivalvular abscess seems to have no relevant impact on 1-year mortality, the rate of readmission or relapse of IE. We suggest, that the higher prevalence of perivalvular abscess in patients undergoing surgery for PVE might have an important impact on the impaired outcome during the first year after surgery for PVE. Therefore, early detection and diagnosis of patients with PVE is crucial and CT imaging should be evaluated in suspected PVE.

\section{Supplementary information}

Supplementary information accompanies this paper at https://doi.org/10. 1186/s12872-020-01338-y.

Additional file 1: Table S1 Perioperative Characteristics. Table S2 Univariate analysis of preoperative risk factors associated with 30-day mortality in patients undergoing surgery for IE

\section{Abbreviations}

AKl: Acute kidney injury; Cl: Confidence interval; CoNS: Coagulase-negative staphylococci; CPB: Cardiopulmonary bypass; ESC: European society of cardiology; IE: Infective endocarditis; IQR: Interquartile range; NVE: Native valve endocarditis; OR: Odds ratio; PVE: Prosthetic valve endocarditis;

SD: Standard deviation

\section{Acknowledgements}

Not applicable.

\section{Authors' contributions}

CW, PBR, TW conceived an supervised the study. CW, PBR, MN, AG, KE, SH, $\mathrm{JM}$ collected and analysed data of patients undergoing surger for infective endocarditis. CW, PBR and TW wrote the article. ACD, AS, EWK, OJL supervised data collection and gave important intellectual support. CW, PB and $\mathrm{SH}$ performed statistical analyses. CW, PBR, MN, AG, KE, JM, ACD, AS,

EWK and OJL performed literature search. All authors revised the manuscript critically and gave final approval.

\section{Funding}

No funding was received.

Availability of data and materials

All data generated and/or analysed during this study are included in this published article and its supplementary files.

\section{Ethics approval and consent to participate}

The study protocol was approved by the institutional review board (Ethics Committee of the Medical Faculty, University of Cologne, 17-407). Individual informed consent was waived due to the retrospective nature of the collected data.

\section{Consent for publication}

Not applicable.

\section{Competing interests}

The authors declare, that they have no competing interests.

\section{Author details}

${ }^{1}$ Department of Cardiothoracic Surgery, University of Cologne, Kerpener Strasse 62, D-50937 Cologne, Germany. ${ }^{2}$ Institute of Medical Statistics and Computational Biology, Faculty of Medicine and University Hospital of Cologne, University of Cologne, Cologne, Germany.

Received: 26 August 2019 Accepted: 9 January 2020

Published online: 03 February 2020

\section{References}

1. Musci M, Hubler M, Amiri A, Stein J, Kosky S, Meyer R, et al. Surgical treatment for active infective prosthetic valve endocarditis: 22-year singleCentre experience. Eur J Cardiothorac Surg. 2010;38:528-38.

2. Romano G, Carozza A, Della Corte A, De Santo LS, Amarelli C, Torella M, et al. Native versus primary prosthetic valve endocarditis: comparison of clinical features and long-term outcome in 353 patients. J Heart Valve Dis. 2004;13:200-8 discussion 8-9.

3. Weber C, Petrov G, Luehr M, Aubin H, Tugtekin SM, Borger MA, et al. Surgical results for prosthetic versus native valve endocarditis: a multicenter analysis. J Thorac Cardiovasc Surg. 2019;(19)32380-3. https://doi.org/10. 1016/j.jtcvs.2019.09.186. [Epub ahead of print]

4. Wang A, Athan E, Pappas PA, Fowler VG Jr, Olaison L, Pare C, et al. Contemporary clinical profile and outcome of prosthetic valve endocarditis. JAMA. 2007;297:1354-61.

5. Habib G, Thuny F, Avierinos JF. Prosthetic valve endocarditis: current approach and therapeutic options. Prog Cardiovasc Dis. 2008;50:274-81.

6. Alonso-Valle H, Farinas-Alvarez C, Garcia-Palomo JD, Bernal JM, Martin-Duran R, Gutierrez Diez JF, et al. Clinical course and predictors of death in prosthetic valve endocarditis over a 20-year period. J Thorac Cardiovasc Surg. 2010;139:887-93.

7. Cahill TJ, Prendergast BD. Infective endocarditis. Lancet. 2016;387:882-93.

8. Grubitzsch H, Tarar W, Claus B, Gabbieri D, Falk V, Christ T. Risks and challenges of surgery for aortic prosthetic valve endocarditis. Heart Lung Circ. 2018;27:333-43

9. Habib G, Lancellotti P, Antunes MJ, Bongiorni MG, Casalta JP, Del Zotti F, et al. 2015 ESC guidelines for the management of infective endocarditis: the 
task force for the Management of Infective Endocarditis of the European Society of Cardiology (ESC). Endorsed by: European Association for CardioThoracic Surgery (EACTS), the European Association of Nuclear Medicine (EANM). Eur Heart J. 2015;36:3075-128.

10. Prendergast $B D$, Tornos P. Surgery for infective endocarditis: who and when? Circulation. 2010;121:1141-52.

11. Hryniewiecki T, Zatorska K, Abramczuk E, Zakrzewski D, Szymanski P, Kusmierczyk M, et al. The usefulness of cardiac $C T$ in the diagnosis of perivalvular complications in patients with infective endocarditis. Eur Radiol. 2019;29:4368-76

12. Gahide G, Bommart S, Demaria R, Sportouch C, Dambia H, Albat B, et al. Preoperative evaluation in aortic endocarditis: findings on cardiac $C T$. AJR Am J Roentgenol. 2010;194:574-8.

13. Li JS, Sexton DJ, Mick N, Nettles R, Fowler VG Jr, Ryan T, et al. Proposed modifications to the Duke criteria for the diagnosis of infective endocarditis. Clin Infect Dis. 2000;30:633-8.

14. Weber C, Gassa A, Rokohl A, Sabashnikov A, Deppe AC, Eghbalzadeh K, et al. Severity of presentation, not sex, increases risk of surgery for infective endocarditis. Ann Thorac Surg. 2019;107(4):1111-7.

15. Edlin P, Westling K, Sartipy U. Long-term survival after operations for native and prosthetic valve endocarditis. Ann Thorac Surg. 2013;95:1551-6.

16. Manne MB, Shrestha NK, Lytle BW, Nowicki ER, Blackstone E, Gordon SM, et al. Outcomes after surgical treatment of native and prosthetic valve infective endocarditis. Ann Thorac Surg. 2012;93:489-93.

17. Edwards MB, Ratnatunga CP, Dore CJ, Taylor KM. Thirty-day mortality and long-term survival following surgery for prosthetic endocarditis: a study from the UK heart valve registry. Eur J Cardiothorac Surg. 1998;14:156-64.

18. Luciani N, Mossuto E, Ricci D, Luciani M, Russo M, Salsano A, et al. Prosthetic valve endocarditis: predictors of early outcome of surgical therapy. A multicentric study. Eur J Cardiothorac Surg. 2017.

19. Lalani T, Chu VH, Park LP, Cecchi E, Corey GR, Durante-Mangoni E, et al. Inhospital and 1-year mortality in patients undergoing early surgery for prosthetic valve endocarditis. JAMA Intern Med. 2013;173:1495-504.

20. Kim YW, Jung SH, Choo SJ, Chung CH, Lee JW, Kim JB. Outcomes of Reoperative valve replacement in patients with prosthetic valve endocarditis: a 20-year experience. Korean J Thorac Cardiovasc Surg. 2018; 51:15-21.

21. Yoshioka D, Toda K, Yokoyama JY, Matsuura R, Miyagawa S, Shirakawa Y, et al. Recent surgical results for active endocarditis complicated with Perivalvular abscess. Circ J. 2017;81:1721-9.

22. Cahill TJ, Baddour LM, Habib G, Hoen B, Salaun E, Pettersson GB, et al. Challenges in infective endocarditis. J Am Coll Cardiol. 2017;69:325-44.

23. Saby L, Laas O, Habib G, Cammilleri S, Mancini J, Tessonnier L, et al. Positron emission tomography/computed tomography for diagnosis of prosthetic valve endocarditis: increased valvular 18F-fluorodeoxyglucose uptake as a novel major criterion. J Am Coll Cardiol. 2013;61:2374-82.

24. Gordon SM, Serkey JM, Longworth DL, Lytle BW, Cosgrove DM 3rd. Early onset prosthetic valve endocarditis: the Cleveland Clinic experience 19921997. Ann Thorac Surg. 2000;69:1388-92.

25. Lopez J, Revilla A, Vilacosta I, Villacorta E, Gonzalez-Juanatey C, Gomez I, et al. Definition, clinical profile, microbiological spectrum, and prognostic factors of early-onset prosthetic valve endocarditis. Eur Heart J. 2007;28:760-5.

26. Fowler VG Jr, Miro JM, Hoen B, Cabell CH, Abrutyn E, Rubinstein E, et al. Staphylococcus aureus endocarditis: a consequence of medical progress. JAMA. 2005:293:3012-21.

\section{Publisher's Note}

Springer Nature remains neutral with regard to jurisdictional claims in published maps and institutional affiliations.

Ready to submit your research? Choose BMC and benefit from:

- fast, convenient online submission

- thorough peer review by experienced researchers in your field

- rapid publication on acceptance

- support for research data, including large and complex data types

- gold Open Access which fosters wider collaboration and increased citations

- maximum visibility for your research: over $100 \mathrm{M}$ website views per year

At $\mathrm{BMC}$, research is always in progress.

Learn more biomedcentral.com/submissions 